\title{
Coating Deposition Using Vacuum Arc and Ablation Metal Plasma
}

\author{
A.I. Ryabchikov, V.M. Matvienko, and I.B. Stepanov \\ Nuclear Physics Institute, 2 a, Lenin ave., Tomsk, 634050, Russia \\ Tel: +7(3822) 42-39-63, Fax: +7(3822) 42-39-34, E-mail: Stepanov@npi.tpu.ru
}

\begin{abstract}
An innovative concept in the development of advanced coating deposition and ion implantation method including an application of filtered DC metal plasma or ablation plasma and highfrequency short-pulsed negative bias voltage with a duty factor in the range $10-99 \%$ are considered.

It was shown that plasma based ion implantation as well as ion-assisted coating deposition may be realized for metal and dielectric samples by variation of negative bias potential in the range of $0-4 \cdot 10^{3} \mathrm{~V}$, pulse repetition rate smoothly adjusted in the range of $(2-4.4) \cdot 10^{5}$ pps and pulse duration in the range of $0.5-2 \mu \mathrm{s}$.

It was experimentally shown that at coating deposition from ablation plasma obtained by high intensity ion beam $\left(j=300 \mathrm{~A} / \mathrm{cm}^{2}, E=350 \mathrm{keV}\right.$, $\tau=90 \mathrm{~ns}$ ) influence on the target, the breakdown of the plasma sheet occurred at de negative bias potential on a substrate more than $60 \mathrm{~V}$. The transfer to $0.5 \mu$ s duration pulses allowed us to increase the bias potential up to $-4 \mathrm{kV}$.
\end{abstract}

\section{Introduction}

Plasma-immersion ion implantation $\left(\mathrm{PI}^{3}\right)$ using gasdischarge plasma was proposed in the works $[1,2]$ and investigated in detail in many other works for both pulsed and continuous plasma generation modes [3-9]. Much less attention has been devoted to the investigations of metal plasma-immersion ion implantation $\left(\mathrm{MPI}^{3}\right)$ [10-12]. Metal plasma-immersion ion implantation and deposition, named as MePIIID [11], was investigated for pulsed metal plasma with rather short bias potential pulse durations $(3-10 \mu \mathrm{s})$ and duty cycle of $10-50 \%$. The regularities of attendant processes for the high-concentration ion implantation regime with surface ion sputtering compensation by plasma deposition using the plasma-immersion approach were investigated in [20-23]. The arc discharge was pulsed with duration from 50 up to $400 \mu$ s. Ion implantation was provided by a dc bias voltage source while the high-concentration ion implantation regime was achieved using bias potential pulse duration less than that for plasma generation. Refs. [10, 13, 14, 17] are devoted to various metal plasma deposition methods with repetitively-pulsed vacuum arc and negative bias potential application. Bias potential pulse duration was less than vacuum-arc pulse duration (several microseconds order of magnitude). The treatment opportunity for internal surfaces of tubes with vacuum-arc source and $\mathrm{PI}^{3}$ application was demonstrated in [18].
It is shown that distribution of ion energy depends greatly on pulse duration.

Different methods of plasma immersion ion implantation have been described in handbook [19] As for $\mathrm{PI}^{3}$ into dielectric materials, the only method using conducting highly transparent mesh electrode [20] has been mentioned. An innovation concept of high frequency short-pulsed bias potential application for metal and dielectric materials treatment has been described in [21-25].

This work is devoted to the investigation of highfrequency short-pulsed plasma immersion ion implantation or deposition (HFSPPI $\left.{ }^{3} \mathrm{D}\right)$ method application to dense vacuum arc metal plasma and very dense ablation plasma deposition technology.

Ablation plasma formed during the influence of high intensity ion and electron beams on the surface of a solid body, presents a considerable interest for many perspective applications. Plasma formed in this way has high density, preserves stoicheometric composition of the target being sputtered and provides record coating deposition rates reaching tens of centimeters per second. Ablation plasma application allows the formation of coatings from various materials, including refractory metals and dielectrics. Unlike the cathode spot or laser evaporation, when plasma source can be considered as a point one, powerful ion beams have an area of cross section equal to tens and hundreds of $\mathrm{cm}^{2}$. Spread of ablation plasma created by pulse intense ion beam (PIIB) is accompanied by a less sharp concentration reduction with an increase of a distance from the target. The degree of ablation plasma ionization depends on both: PIIB parameters and target characteristics and can vary from the part of percent to $100 \%$.

\section{Physical model of HFSPPI ${ }^{3} D$ method application for ablation plasma deposition}

It is proposed that plasma flow, formed by vacuum arc or pulsed intense ion beam interaction with a target surface, moves towards a sample holder with velocity $v \sim(1-2) \cdot 10^{4} \mathrm{~m} / \mathrm{s}$. Though PIIB duration usually does not exceed several hundreds of nanoseconds, the duration of the plasma flow formed as a result of the influence on the target constitutes several tens of microseconds. Dielectric, semiconducting and conducting samples to be treated are mounted on a metal holder. The physics of the processes, providing the formation of ion streams, differs for conductive and dielectric materials. When metallic samples are used, the load of a pulsed bias potential generator has both 
capacitive and active components. The capacitive component has a place only at a period of transition, when the plasma sheath enlarges up to the stationary state defined by the Child-Langmuir limit or by a fixed distance between samples and plasma-emission boundary (in case of plasma-emission boundary limitation by grid electrode). Immersion ion implantation from vacuum arc plasma into metallic substrates, taking into account an active constituent of pulse generator load and the existence of plasma flow direct velocity, could be realized using dc, long-pulsed and shortpulsed bias potential at the holder and accordingly on the sample surface. According to investigations, in the case of ablation plasma it is practically impossible to use dc bias potential more than 50-60 V.

The situation changes when dielectric samples are used. If a dielectric sample fully covers the sample holder, then the active component of load is completely excluded and ion accelerating processes are determined by the thickness and permittivity of the dielectric, by the dynamics of ion accumulation on the surface of the sample, and by plasma and bias potential pulse parameters. If the bias potential pulse is long and the plasma density is high, then charging of the dielectric can complete quickly enough so that all the electric field will be concentrated only inside of a capacitor composed of the dielectric charged surface and the potential electrode (holder). After that, there will be no electric field outside of this capacitor, so it is unsuitable to use bias potential pulses with duration greater than the dielectric surface charging time. Hence, it is important to determine the parameters of the plasma, sample and bias potential pulse required to realize energy-optimal regimes of $\mathrm{PI}^{3}$ using dense plasma. If pulse duration of the bias potential is less than that of ablation plasma, an electric field arises between the plasma and the charged surface of dielectric sample after switching off the bias potential on the holder. As a result, not ions, but electrons, are extracted from plasma. The current of electrons and their mobility are much greater then the current and mobility of ions, so the surface charge compensation will take place almost instantly. Estimates show that charge compensation time does not exceed a nanosecond. This compensation times of great importance since, in practice. It determines the allowable pulse duty factor for $\operatorname{HFSPPI}^{3} \mathrm{D}$ regimes realization. Using the microsecond pulse duration and possible pause duration, for example, allows one to increase the pulse duty factor up to unity. On the other hand, it means that for high $f_{\tau}$ values (close to 1) not only plasma deposition, but also conventional and high-concentration ion implantation mode may be realized.

Vacuum arc and ablation plasma differs by plasma concentration by several orders. Concentration of the vacuum arc plasma filtered from microparticles usually does not exceed $5 \cdot 10^{10} \mathrm{~cm}^{-3}$. Concentration of the ablation plasma on the distances used for the coatings deposition $(10-40 \mathrm{~cm})$ is $10^{12}-10^{14} \mathrm{~cm}^{-3}$. It means that plasma sheath with the thickness of fractions of millimeters will be formed at bias potential supply on the target. Electric field strength in a plasma sheath and especially on the bias potential electrodes (samples) surface will be determined not only by classical $U_{b} / d$ ( $d$ is the sheath thickness) but by the surface microrelief. Obviously, one should expect a considerable increase in the electric field strength on microasperities and roughness of the sample surface. Increase in the electric field strength should be accompanied by the increase in the electron current and a sufficient probability of the cathode spot appearance. When cathode spot appears, plasma formed by them propagates from the surface of a negative electrode at the rate of $\sim(1-2) \cdot 10^{6} \mathrm{~cm} / \mathrm{s}$. Plasma sheath thickness and propagation rate of plasma torch determine the time, after which the shortening of the charge division layer and consequently breakdown of plasma sheath will take place. That is why long bias potential pulses or dc bias potential application has no perspectives for the improvement of coatings formed by ablation plasma.

\section{Experimental installations}

Experimental installation for vacuum arc filtered plasma deposition presents a vacuum chamber with $0.5 \mathrm{~m}^{3}$ volume, on which a vacuum arc plasma generator equipped with plasma filter for microparticles removal is installed [21]. Metal and dielectric samples were placed on the conductive holder both in case of application of vacuum arc plasma and ablation plasma. High frequency short pulse bias potentials were applied to the holder.

A schematic of the experiment on thin film deposition using PIIB is shown in Fig. 1.

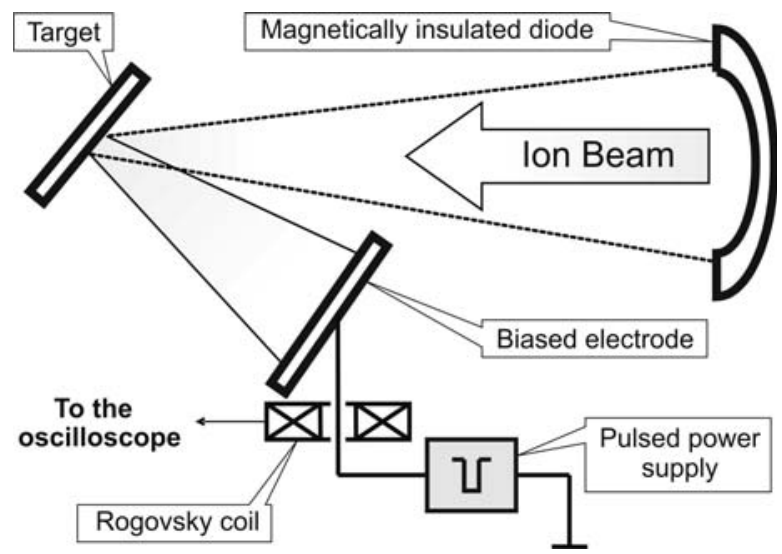

Fig. 1. A schematic of the experiment on thin film deposition using PIIB

To produce pulsed ion beams having energy of $300 \mathrm{keV}$ and current density of up to $200 \mathrm{~A} / \mathrm{cm}^{2}$, a magnetically insulated diode is used in the TEMP accelerator in a magnetic-insulation mode applying an external pulsed transverse magnetic field [26]. The major advantages of this type of diode over other diode systems are long service life and ion beam pa- 
rameter stability. The ground electrode of the magnetically-insulated diode (MID) has the shape of an open coil, one end of which is connected to the vacuum chamber. The electrodes are $40 \mathrm{~mm}$ wide, and their working portion is $200 \mathrm{~mm}$ long. The high potential electrode is made from graphite. The nanosecond generator of the accelerator forms two high-voltage pulses of opposite polarities having amplitudes of 150 and $300 \mathrm{kV}$. The first high voltage pulse is used to create explosion plasma and the second one of negative polarity for ion acceleration. The beam composition depends mainly on the material of the high potential electrode and on the state of the graphite surface. The beam generated is composed of carbon ions and protons (70 and $30 \%$, respectively).

The ion beam formed in a magnetically-insulated diode [26], was focused on the Ti target. Current density in the focus of ion beam on the target constituted $300 \mathrm{~A} / \mathrm{cm}^{2}$ with energy of hydrogen and carbon ions up to $350 \mathrm{keV}$ and pulse duration of $90 \mathrm{~ns}$. The target was located under the angle to an incident beam in such a way, that the steam-plasma torch spreading along the normal to the target surface fell onto potential electrode, placed at the distance of $10 \mathrm{~cm}$ from the target being sputtered.

\section{Experimental results and discussions}

The adhesion strength investigations of the Ti coatings deposited using vacuum arc plasma on glass-ceramic and stainless steel substrates using HFSPPI ${ }^{3} \mathrm{D}$ method showed their significant improvement with bias potential amplitude increase. All coatings were deposited using a duty factor of 0.42 . The coating delamination critical force (Fig. 2) increases from $0.5 \mathrm{~N}$ for coatings deposited on a metal sample without biasing, to $1.2 \mathrm{~N}$ for the case when bias voltage amplitude was $-500 \mathrm{~V}$.



Fig. 2. Ti coating delamination critical force depending on bias potential pulse. Duty factor is 0.42

Further bias potential amplitude increase results in a small improvement of the coating adhesion strength up to $1.23 \mathrm{~N}$ at $-2000 \mathrm{~V}$. Coating deposited on the glass-ceramic sample demonstrated the same behavior. Corresponding values for it were $0.3 \mathrm{~N}$ for deposi- tion without biasing, $1.16 \mathrm{~N}$ for bias potential amplitude $-500 \mathrm{~V}$ and $1.18 \mathrm{~N}$ in a higher amplitude range.

Experiments with ablation plasma showed the following.

Investigations with the dc bias potential application showed that their stable use (without breakdown) is possible with amplitudes not exceeding - (50-60) V.

In the experiments with short-pulse bias potential, the generator with the potential amplitude regulated in the range of $0-4 \cdot 10^{3} \mathrm{~V}$, pulse repetition rate smoothly adjusted in the range of $2-4.4 \cdot 10^{5} \mathrm{pps}$ and pulse duration ranging from 0.5 to $3 \mu$ s was used as a negative bias potential source.

Figure 3 presents oscillograms of the current in the collector circuit during bias potential application to the sample with pulse duration of $3 \mu$ s and pulse repetition rate of $1.6 \cdot 10^{5} \mathrm{pps}$.

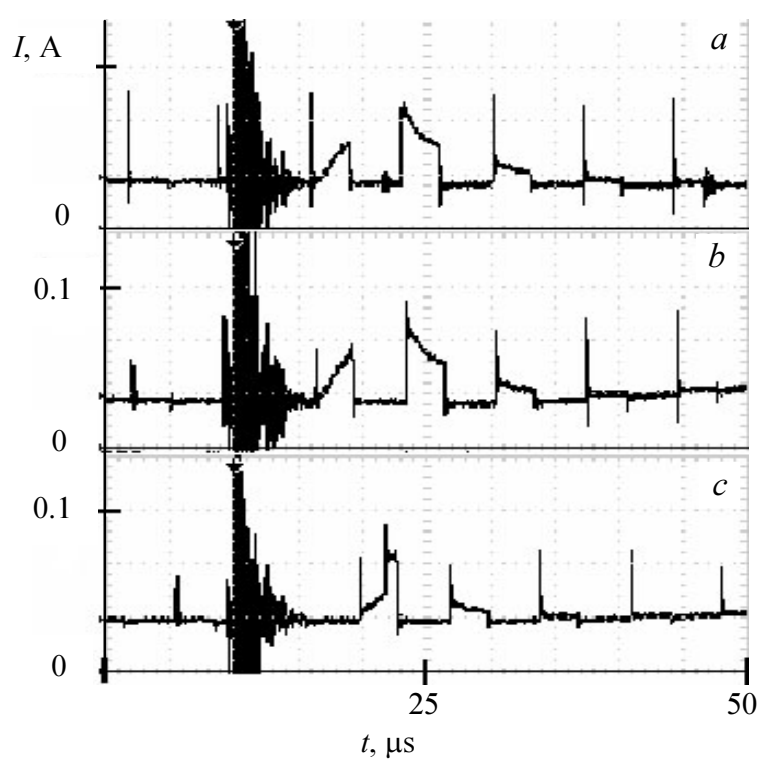

Fig. 3. High frequency short pulse negative bias voltage application for ablation plasma deposition. $\tau_{b}=3 \mu \mathrm{s}$; $f=1.6 \cdot 10^{5}$ pps: $U_{\text {bias }}=-100(a) ;-250(b) ;-500 \mathrm{~V}(c)$

It follows from the presented data that the current amplitude of various pulses clearly determines the moment of plasma reaching the collector and the dynamics of changing its concentration in time. Total plasma duration constitutes 20-30 $\mu$ s. Time of plasma reaching the sample after the accelerator shot is about $10 \mu \mathrm{s}$. Thus, the plasma propagation velocity makes $\sim 10^{6} \mathrm{~cm} / \mathrm{s}$. With bias potentials to $250 \mathrm{~V}$ (Figs. 3, a and $b$ ) current pulses prove to be sufficiently smooth and no breakdowns are observed. Oscillations indicating the breakdown appearance are observed when the bias potential pulse amplitude increases to $500 \mathrm{~V}$ (Fig. 3, c). It is typical, that the breakdown takes place not from the very beginning of the bias pulse (see Fig. 3,c).

Taking into account this effect, the bias potential pulse duration was decreased to $1 \mu \mathrm{s}$ in the following series of experiments. The characteristic oscillograms of the current pulses are shown in Fig. 4 for this case. 




Fig. 4. High frequency short pulse negative bias voltage application for ablation plasma deposition: $\tau_{b}=1 \mu \mathrm{s}$;

$$
U_{\text {bias }}=-2000 \mathrm{~V}
$$

The oscillogram analysis shows that even the potential amplitude increase to $-2 \mathrm{kV}$ does not cause any obvious breakdown during the bias pulse. The subsequent decrease in the bias potential pulse duration to $0.5 \mu \mathrm{s}$ enabled to increase the bias potential amplitude to $4 \mathrm{kV}$. Investigation results of a Ti coating deposited using ablation plasma delamination critical force depending on bias potential amplitude is shown in Fig. 2. It is obvious that the coating adhesion increases with the increase in the bias potential amplitude in both metal and glass ceramic samples.

\section{Conclusions}

Thus, the investigations performed allow making a conclusion about the possibility of amplitude sufficient bias potential application for the ion-assisted deposition of dense vacuum arc metal plasma and ablation plasma. In the case of ablation plasma application, the pulse duration must be shorter than $1 \mu$ s.

It is obvious that in order to provide energetic efficiency of such bias potentials it is necessary to increase the pulse repetition rate to the value providing effective ion mixing of coatings deposition. Taking into account, that ablation plasma can provide sufficient currents to hundreds ampere, the design of the generator operating in dc mode seems inexpedient and difficult to realize. At the same time, the generator capable of formation of the bias potential burst of pulses with the specified duration and frequency and intended for ablation plasma pulse duration can be realized.

\section{References}

[1] J. Conrad, J. Appl. Phys. 62, 777 (1987).

[2] J.R. Conrad, R.A. Dodd, F.J. Worzala, and X. Qui, Surf. Coat. Technol. 36, 927 (1988).
[3] T. Sheng, S.B. Felch, and C.B. Cooper, J. Vac. Sci. Technol. B12 969 (1994).

[4] J.B. Liu, S.S. Iyer, R. Gronsky, C. Hu, and N.W. Cheung, Appl. Phys. Lett. 67, 2361(1995).

[5] M. Nastasi, A.A. Elmoursi, R.J. Faehl et al., Mater. Res. Soc. Symp. Proc. 396, 455 (1996).

[6] K. Holmberg and A. Matthews, Coatings Tribology: Properties, Techniques and Applications in Surface Engineering, Amsterdam, Elsevier, 1994, p. 53

[7] S.-H. Lin, B.J. Feldman, and D. Li, Appl. Phys. Lett. 69, 2373 (1996).

[8] K.C. Walter, M. Nastasi, N.P. Baker et al., Surf. Coat. Technol. 103-104, 205 (1998).

[9] R. Gunzel, J. Brutscher, S. Mandl, and W. Moller, Surf. Coat. Technol. 96, 16 (1997).

[10] I.G. Brown, A. Anders, M.R. Dickinson, R.A. MacGill, and O.R. Monteiro, Surf. Coat. Technol. 112, 271 (1999).

[11] I.G. Brown, X. Godechot, and K.M. Yu, Appl. Phys. Lett. 58, 1392 (1991).

[12] Bin Liu, B.Y. Jiang, Y. Fu, D.J. Cheng, X.F. Wu, and S.Z. Yang, Thin Solid Films 349, 110 (1999).

[13] A.I. Ryabchikov, R.A. Nasyrov, and A.A. Kompaniets, in Proc. of the Int. Conf. on Ion Implantation and Ion Beam Equipment, 1990, p. 118.

[14] A.I. Ryabchikov and R.A. Nasyrov, Nucl. Instrum Methods. Phys. Res. B61, 48 (1991).

[15] A.I. Ryabchikov and R.A. Nasyrov, Poverhnost' 3, 98 (1992)

[16] A.I. Ryabchikov, S.V. Dektyarev, and I.B. Stepanov, Izv. Vyssh. Uchebn. Zaved. Fiz. 2, 82 (1994).

[17] T. Zhang, B.Y. Tang, Z.M. Zeng et al., Surf. Coat. Technol. 128-129, 231 (2000).

[18] A.I. Ryabchikov, Diss. Doct. Ph.-Mat. Sc., Tomsk, Russia, 1993.

[19] A. Anders, Handbook of Plasma Immersion Ion Implantation and Deposition, John Wiley and Sons, Inc., 2000

[20] J.N. Matossian, R.N. Schumacher, and D.M. Pepper, U.S. patent 5,374,456, CA, 1994

[21] A.I. Ryabchikov, I.A. Ryabchikov, and I.B. Stepanov, Vacuum 445-449, 78 (2005).

[22] A.I. Ryabchikov and I.A. Ryabchikov, in Proc. of the $6^{\text {th }}$ Int. Conf. on Modification of Materials with Particle Beams and Plasma Flows, 2002, p. 571582.

[23] A.I. Ryabchikov, I.A. Ryabchikov, and I.B. Stepanov, Vacuum 331-336, 78 (2005).

[24] A.I. Ryabchikov, I.A. Ryabchikov, I.B. Stepanov, and Yu.P. Usov, Surf. Coat. Technol. 201, 65236525 (2007).

[25] A.I. Ryabchikov, I.A. Ryabchikov, and I.B. Stepanov, Surf. Coat. Technol. 201, 8610-8614 (2007).

[26] V.K. Struts, A.N. Zakoutaev, V.M. Matvienko et al., Surf. Coat. Technol. 494, 158 (2002). 